\title{
ON THE PERIODIC SOLUTIONS OF A RIGID DUMBBELL SATELLITE IN A CIRCULAR ORBIT
}

\author{
JUAN L.G. GUIRAO ${ }^{1}$, JUAN A. VERA ${ }^{2}$ AND BRUCE A. WADE ${ }^{3}$
}

\begin{abstract}
The aim of the present paper is to provide sufficient conditions for the existence of periodic solutions of the perturbed attitude dynamics of a rigid dumbbell satellite in a circular orbit.
\end{abstract}

\section{Introduction AND STATEMENT OF the MAIN RESUlts}

In this paper we consider the attitude dynamics, perturbed by small torques, of a rigid dumbbell satellite in a circular orbit under the gravitational torque of a central Newtonian force field. Our objective is to provide sufficient conditions for the existence of periodic motions about the satellite's center of mass that are asymptotic to translational motion in an absolute coordinate system. This type of motion, denoted, cylindrical equilibrium, is well known in the astrophysics literature on satellite's dynamics (see for instance [4, 7, 8]).

These motions have an important application to satellite orientation problems because a satellite can reach some specified nominal regime along periodic trajectories only through the influence of gravitational torques and other small perturbed torques induced by some control mechanism.

Following the methods developed in [8], the equations of motion governing the attitude dynamics of a rigid dumbbell satellite are

$$
\begin{aligned}
& \frac{d^{2} \theta}{d t^{2}}-2 \frac{d \phi}{d t}\left(1+\frac{d \theta}{d t}\right) \tan \phi+3 \sin \theta \cos \theta=\varepsilon F_{1}^{*}\left(t, \theta, \frac{d \theta}{d t}, \phi, \frac{d \phi}{d t}\right) \\
& \frac{d^{2} \phi}{d t^{2}}+\left(\left(1+\frac{d \theta}{d t}\right)^{2}+3 \cos ^{2} \theta\right) \sin \phi \cos \phi=\varepsilon F_{2}^{*}\left(t, \theta, \frac{d \theta}{d t}, \phi, \frac{d \phi}{d t}\right)
\end{aligned}
$$

with $\theta$ and $\phi$ the eulerian angles of nutation and precession. The perturbed torques $F_{i}^{*}$, are smooth functions periodic in the variable $t$ with

$$
F_{1}^{*}\left(t, 0, \frac{d \theta}{d t}, 0, \frac{d \phi}{d t}\right) \equiv 0, \quad F_{2}^{*}\left(t, 0, \frac{d \theta}{d t}, 0, \frac{d \phi}{d t}\right) \equiv 0,
$$

and $\varepsilon$ a small real parameter. In this work we are interesed in the periodic

\footnotetext{
Key words and phrases. Dumbbell satellite, periodic orbits, averaging theory.

2010 Mathematics Subject Classification. Primary: 70E17, 70E20, 70E40. Secondary:
} $37 \mathrm{C} 27$. 
functions emerging from the equilibrium solution $\theta=0$ and $\phi=0$ of (11) when $\varepsilon \rightarrow 0$.

By means of the change of coordinates $x=\theta$ and $y=\phi$ and linearizing the equations of (11) in this equilibrium we obtain

$$
\begin{aligned}
& \frac{d^{2} x}{d t^{2}}+3 x=\varepsilon F_{1}\left(t, x, \frac{d x}{d t}, y, \frac{d y}{d t}\right)+\varepsilon^{2} R_{1}\left(t, x, \frac{d x}{d t}, y, \frac{d y}{d t}, \varepsilon\right) \\
& \frac{d^{2} y}{d t^{2}}+4 y=\varepsilon F_{2}\left(t, x, \frac{d x}{d t}, y, \frac{d y}{d t}\right)+\varepsilon^{2} R_{2}\left(t, x, \frac{d x}{d t}, y, \frac{d y}{d t}, \varepsilon\right)
\end{aligned}
$$

with

$$
\begin{aligned}
& F_{1}\left(t, x, \frac{d x}{d t}, y, \frac{d y}{d t}\right)=f_{1}\left(t, \frac{d x}{d t}, \frac{d y}{d t}\right) x+f_{2}\left(t, \frac{d x}{d t}, \frac{d y}{d t}\right) y \\
& F_{2}\left(t, x, \frac{d x}{d t}, y, \frac{d y}{d t}\right)=f_{3}\left(t, \frac{d x}{d t}, \frac{d y}{d t}\right) x+f_{4}\left(t, \frac{d x}{d t}, \frac{d y}{d t}\right) y
\end{aligned}
$$

and $f_{i}$ smooth functions in the variables $\left(t, \frac{d x}{d t}, \frac{d y}{d t}\right)$. On the other hand the functions $f_{i}$ are periodic in $t$ and in resonance $p: q$ with some of the periodic solutions of the unperturbed dumbbell satellite given by

$$
\begin{aligned}
& \frac{d^{2} x}{d t^{2}}+3 x=0 \\
& \frac{d^{2} y}{d t^{2}}+4 y=0 .
\end{aligned}
$$

The objective of this work is to provide, using as a main tool the averaging theory, a system of nonlinear equations whose simple zeros provide periodic solutions of the perturbed dumbbell satellite in circular orbit with equations of motion given by (2). Some other works using similar techniques (see Llibre et al. [1] for more details), are [2, 3] or [4] where the method is used for action angle variables. In order to present our results we need some preliminary definitions and notations.

The unperturbed system (3) has a unique singular point, the origin with eigenvalues

$$
\pm \sqrt{3} i, \quad \pm 2 i
$$

Consequently this system in the phase space $\left(x, \frac{d x}{d t}, y, \frac{d y}{d t}\right)$ has two planes filled with periodic solutions with the exception of the origin. These periodic solutions have periods

$$
T_{1}=\frac{2 \pi}{\sqrt{3}} \quad \text { or } \quad T_{2}=\pi
$$

according to whether they belong to the plane associated to the eigenvectors with eigenvalues $\pm \sqrt{3} i$ or $\pm 2 i$, respectively. We shall study which of these periodic solutions persist for the perturbed system (2) when the parameter 
$\varepsilon$ is sufficiently small and the perturbed functions $F_{i}$ for $i=1,2$ have period either $p T_{1} / q$, or $p T_{2} / q$, where $p$ and $q$ are positive integers relatively prime.

We define the functions:

$$
\begin{aligned}
& \mathcal{F}_{1}\left(X_{0}, Y_{0}\right)=\frac{1}{2 p \pi} \int_{0}^{p T_{1}} \sin (\sqrt{3} t) \Delta_{1}(t) f_{1}\left(t, \Delta_{2}(t), 0\right) d t \\
& \mathcal{F}_{2}\left(X_{0}, Y_{0}\right)=\frac{\sqrt{3}}{2 p \pi} \int_{0}^{p T_{1}} \cos (\sqrt{3} t) \Delta_{1}(t) f_{1}\left(t, \Delta_{2}(t), 0\right) d t \\
& \mathcal{G}_{1}\left(Z_{0}, W_{0}\right)=\frac{1}{p \pi} \int_{0}^{p T_{2}} \sin (2 t) \Delta_{3}(t) f_{4}\left(t, 0, \Delta_{4}(t)\right) d t \\
& \mathcal{G}_{2}\left(Z_{0}, W_{0}\right)=\frac{2}{p \pi} \int_{0}^{p T_{2}} \cos (2 t) \Delta_{3}(t) f_{4}\left(t, 0, \Delta_{4}(t)\right) d t
\end{aligned}
$$

with

$$
\begin{aligned}
& \Delta_{1}(t)=X_{0} \cos (\sqrt{3} t)+\frac{Y_{0}}{\sqrt{3}} \sin (\sqrt{3} t) \\
& \Delta_{2}(t)=Y_{0} \cos (\sqrt{3} t)-\sqrt{3} X_{0} \sin (\sqrt{3} t) \\
& \Delta_{3}(t)=Z_{0} \cos (2 t)+\frac{W_{0}}{2} \sin (2 t) \\
& \Delta_{4}(t)=W_{0} \cos (2 t)-2 Z_{0} \sin (2 t)
\end{aligned}
$$

A zero $\left(X_{0}^{*}, Y_{0}^{*}\right)$ of the nonlinear system

$$
\mathcal{F}_{1}\left(X_{0}, Y_{0}\right)=0, \quad \mathcal{F}_{2}\left(X_{0}, Y_{0}\right)=0
$$

such that

$$
\operatorname{det}\left(\left.\frac{\partial\left(\mathcal{F}_{1}, \mathcal{F}_{2}\right)}{\partial\left(X_{0}, Y_{0}\right)}\right|_{\left(X_{0}, Y_{0}\right)=\left(X_{0}^{*}, Y_{0}^{*}\right)}\right) \neq 0
$$

is called a simple zero of system (5). Similarly, a zero $\left(Z_{0}^{*}, W_{0}^{*}\right)$ of the nonlinear system

$$
\mathcal{G}_{1}\left(Z_{0}, W_{0}\right)=0, \quad \mathcal{G}_{2}\left(Z_{0}, W_{0}\right)=0
$$

such that

$$
\operatorname{det}\left(\left.\frac{\partial\left(\mathcal{G}_{1}, \mathcal{G}_{2}\right)}{\partial\left(Z_{0}, W_{0}\right)}\right|_{\left(Z_{0}, W_{0}\right)=\left(Z_{0}^{*}, W_{0}^{*}\right)}\right) \neq 0,
$$

is called a simple zero of system (66).

Our main results on the periodic solutions of the perturbed dumbbell satellite (2) are the following.

Theorem 1. Assume that the function $F_{1}^{*}$ and $F_{2}^{*}$ of the perturbed dumbbell satellite with equations of motion (1) are periodic in $t$ of period $p T_{1} / q$ with $p$ and $q$ positive integers relatively prime. Then, for $\varepsilon \neq 0$ sufficiently small and for every simple zero $\left(X_{0}^{*}, Y_{0}^{*}\right) \neq(0,0)$ of the nonlinear system (5), the perturbed dumbbell satellite (1) has a periodic solution 


$$
\begin{aligned}
(\theta(t, \varepsilon), & \left.\frac{d \theta}{d t}(t, \varepsilon), \phi(t, \varepsilon), \frac{d \phi}{d t}(t, \varepsilon)\right) \text { with } \\
& \lim _{\varepsilon \rightarrow 0}\left(\theta(0, \varepsilon), \frac{d \theta}{d t}(0, \varepsilon), \phi(0, \varepsilon), \frac{d \phi}{d t}(0, \varepsilon)\right)=\left(X_{0}^{*}, Y_{0}^{*}, 0,0\right) .
\end{aligned}
$$

Theorem 11 is proved in section [2, Its proof is based in the averaging theory for computing periodic solutions, see the Appendix of the paper.

We provide an application of Theorem 1 in the following corollary, which will be proved in section 3 ,

Corollary 2. We consider the system (1) with

$$
\begin{aligned}
F_{1}^{*}\left(t, \theta, \frac{d \theta}{d t}, \phi, \frac{d \phi}{d t}\right) & =\sin \theta\left(\frac{d \theta}{d t}\right)^{4}+\sin \phi \sin \theta\left(1-\left(\frac{d \phi}{d t}\right)^{2}\right), \\
F_{2}^{*}\left(t, \theta, \frac{d \theta}{d t}, \phi, \frac{d \phi}{d t}\right) & =\cos \theta-\sin (\sqrt{3} t) \sin \theta \frac{d \theta}{d t}-\sin \theta\left(\frac{d \theta}{d t}\right)^{2} \\
& -\cos \phi\left(1-\left(\frac{d \phi}{d t}\right)^{2}\right) .
\end{aligned}
$$

Then, for $\varepsilon \neq 0$ sufficiently small the system (1) has one periodic solution with

$$
\lim _{\varepsilon \rightarrow 0}\left(\theta(0, \varepsilon), \frac{d \theta}{d t}(0, \varepsilon), \phi(0, \varepsilon), \frac{d \phi}{d t}(0, \varepsilon)\right)=\left(\frac{\sqrt{3}}{3}, 0,0,0\right) .
$$

Similarly we obtain the following result.

Theorem 3. Assume that the functions $F_{1}^{*}$ and $F_{2}^{*}$ of the perturbed dumbbell satellite with equations of motion (1) are periodic in $t$ of period $p T_{2} / q$ with $p$ and $q$ positive integers, relatively prime. Then for $\varepsilon \neq 0$ sufficiently small and for every simple zero $\left(Z_{0}^{*}, W_{0}^{*}\right) \neq(0,0)$ of the nonlinear system (6), the perturbed dumbbell satellite (1) has a periodic solution

$$
\begin{aligned}
(\theta(t, \varepsilon), & \left.\frac{d \theta}{d t}(t, \varepsilon), \phi(t, \varepsilon), \frac{d \phi}{d t}(t, \varepsilon)\right) \text { with } \\
& \lim _{\varepsilon \rightarrow 0}\left(\theta(0, \varepsilon), \frac{d \theta}{d t}(0, \varepsilon), \phi(0, \varepsilon), \frac{d \phi}{d t}(0, \varepsilon)\right)=\left(0,0, Z_{0}^{*}, W_{0}^{*}\right) .
\end{aligned}
$$

The proof of Theorem 3 is analogous Theorem 1 .

On the other hand, we provide an application of Theorem 3 in the next corollary, which will be proved in section 3 .

Corollary 4. We consider the system (11) with

$$
\begin{aligned}
& F_{1}^{*}\left(t, \theta, \frac{d \theta}{d t}, \phi, \frac{d \phi}{d t}\right)=\sin \phi \sin \theta \frac{d \phi}{d t}+\sin \phi+\sin (2 t) \sin \phi\left(1-\frac{d \phi}{d t}\right) \frac{d \phi}{d t}, \\
& F_{2}^{*}\left(t, \theta, \frac{d \theta}{d t}, \phi, \frac{d \phi}{d t}\right)=\sin \phi-\sin (2 t) \sin \phi \frac{d \phi}{d t}-\sin \phi\left(\frac{d \phi}{d t}\right)^{2} .
\end{aligned}
$$


Then, for $\varepsilon \neq 0$ sufficiently small, the system (1) has three periodic solutions with

$$
\begin{aligned}
& \lim _{\varepsilon \rightarrow 0}\left(\theta(0, \varepsilon), \frac{d \theta}{d t}(0, \varepsilon), \phi(0, \varepsilon), \frac{d \phi}{d t}(0, \varepsilon)\right)=\left(0,0,1, \frac{2 \sqrt{3}}{3}\right), \\
& \lim _{\varepsilon \rightarrow 0}\left(\theta(0, \varepsilon), \frac{d \theta}{d t}(0, \varepsilon), \phi(0, \varepsilon), \frac{d \phi}{d t}(0, \varepsilon)\right)=\left(0,0, \frac{1-\sqrt{17}}{4}, 0\right), \\
& \lim _{\varepsilon \rightarrow 0}\left(\theta(0, \varepsilon), \frac{d \theta}{d t}(0, \varepsilon), \phi(0, \varepsilon), \frac{d \phi}{d t}(0, \varepsilon)\right)=\left(0,0, \frac{1+\sqrt{17}}{4}, 0\right) .
\end{aligned}
$$

Remark 1. The momenta apply to a rigid dumbbell satellite in a circular orbit under the gravitational torque of a central Newtonian force field are, in general, functions of the Euler's angle and some maps which depend of the independent variable $t$ (time). The applications presented in Corollaries (2) and 4 represent some usual momenta which model the solar radiation obtained by the solar panels located at the satellite, see for more information [5].

\section{Proof of Theorems 1 and 3}

Introducing the variables $(X, Y, Z, W)=\left(x, \frac{d x}{d t}, y, \frac{d y}{d t}\right)$ we write the differential system of the perturbed dumbbell satellite (2) as a first-order differential system defined in $\mathbb{R}^{4}$, as follows:

$$
\begin{aligned}
& \frac{d X}{d t}=Y, \\
& \frac{d Y}{d t}=-3 X+\varepsilon F_{1}(t, X, Y, Z, W)+\varepsilon^{2} R_{1}(t, X, Y, Z, W, \varepsilon), \\
& \frac{d Z}{d t}=W, \\
& \frac{d W}{d t}=-4 Z+\varepsilon F_{2}(t, X, Y, Z, W)+\varepsilon^{2} R_{2}(t, X, Y, Z, W, \varepsilon) .
\end{aligned}
$$

System (7) with $\varepsilon=0$ is equivalent to the unperturbed dumbbell satellite system (3). On the other hand, the periodic orbits of the unperturbed system are described in the following lemma.

Lemma 5. The periodic solutions of the unperturbed system with $\varepsilon=0$ are

$$
\begin{aligned}
& X(t)=X_{0} \cos (\sqrt{3} t)+\frac{Y_{0}}{\sqrt{3}} \sin (\sqrt{3} t), \\
& Y(t)=Y_{0} \cos (\sqrt{3} t)-\sqrt{3} X_{0} \sin (\sqrt{3} t), \\
& Z(t)=0, \\
& W(t)=0,
\end{aligned}
$$


of period $T_{1}$, and

$$
\begin{aligned}
& X(t)=0, \\
& Y(t)=0, \\
& Z(t)=Z_{0} \cos (2 t)+\frac{W_{0}}{2} \sin (2 t), \\
& W(t)=W_{0} \cos (2 t)-2 Z_{0} \sin (2 t),
\end{aligned}
$$

of period $T_{2}$.

Proof. Since the unperturbed system is a linear differential system, the proof is routine.

Proof of Theorem 1. Assume that the function $F_{1}$ and $F_{2}$ of the dumbbell satellite system with equations of motion (2) are periodic in $t$ of period $p T_{1} / q$ with $p$ and $q$ positive integers, relatively prime. Then system (2) is periodic in $t$ with period $p T_{1}$.

We shall apply Theorem 6 of the appendix to the differential system (7). We note that system (7) can be written as system (11), taking

$$
\begin{gathered}
\mathbf{x}=\left(\begin{array}{c}
X \\
Y \\
Z \\
W
\end{array}\right), G_{0}(t, \mathbf{x})=\left(\begin{array}{c}
Y \\
-3 X \\
W \\
-4 Z
\end{array}\right), \\
G_{1}(t, \mathbf{x})=\left(\begin{array}{c}
F_{1}(t, X, Y, Z, W) \\
0 \\
F_{2}(t, X, Y, Z, W)
\end{array}\right)
\end{gathered}
$$

and

$$
G_{2}(t, \mathbf{x}, \varepsilon)=\left(\begin{array}{c}
0 \\
\varepsilon^{2} R_{1}(t, X, Y, Z, W, \varepsilon) \\
0 \\
\varepsilon^{2} R_{2}(t, X, Y, Z, W, \varepsilon)
\end{array}\right) .
$$

We shall study which periodic solutions (8) of the unperturbed system corresponding to the system (77) with $\varepsilon=0$ can be continued to periodic solutions of the unperturbed system for $\varepsilon \neq 0$ sufficiently small.

We shall describe the different elements which appear in the statement of Theorem 6. Thus we have that $\Omega=\mathbb{R}^{4}, k=2$ and $n=4$. Let $r_{1}>0$ be arbitrarily small and let $r_{2}>0$ be arbitrarily large. We take the open and bounded subset $V$ of the plane $Z=W=0$ as

$$
V=\left\{\left(X_{0}, Y_{0}, 0,0\right) \in \mathbb{R}^{4}: r_{1}<\sqrt{X_{0}^{2}+Y_{0}^{2}}<r_{2}\right\}
$$


As usual $\mathrm{Cl}(V)$ denotes the closure of $V$. If $\alpha=\left(X_{0}, Y_{0}\right)$, then we can identify $V$ with the set

$$
\left\{\alpha \in \mathbb{R}^{2}: r_{1}<\|\alpha\|<r_{2}\right\},
$$

here $\|\cdot\|$ denotes the Euclidean norm of $\mathbb{R}^{2}$. The function $\beta: \mathrm{Cl}(V) \rightarrow \mathbb{R}^{2}$ is $\beta(\alpha)=(0,0)$. Therefore, in our case the set

$$
\mathcal{Z}=\left\{\mathbf{z}_{\alpha}=(\alpha, \beta(\alpha)), \alpha \in \mathrm{Cl}(V)\right\}=\left\{\left(X_{0}, Y_{0}, 0,0\right) \in \mathbb{R}^{4}: r_{1} \leq \sqrt{X_{0}^{2}+Y_{0}^{2}} \leq r_{2}\right\} .
$$

Clearly for each $\mathbf{z}_{\alpha} \in \mathcal{Z}$ we can consider the periodic solution $\mathbf{x}\left(t, \mathbf{z}_{\alpha}\right)=$ $(X(t), Y(t), 0,0)$ given by (8) of period $p T_{1}$.

Computing the fundamental matrix $M_{\mathbf{z}_{\alpha}}(t)$ of the linear differential system with $\varepsilon=0$ associated to the $T$-periodic solution $\mathbf{z}_{\alpha}=\left(X_{0}, Y_{0}, 0,0\right)$ such that $M_{\mathbf{z}_{\alpha}}(0)$ is the identity of $\mathbb{R}^{4}$, we conclude that $M(t)=M_{\mathbf{z}_{\alpha}}(t)$ is equal to

$$
\left(\begin{array}{cccc}
\cos (\sqrt{3} t) & \frac{\sin (\sqrt{3} t)}{\sqrt{3}} & 0 & 0 \\
-\sqrt{3} \sin (\sqrt{3} t) & \cos (\sqrt{3} t) & 0 & 0 \\
0 & 0 & \cos (2 t) & \frac{\sin (2 t)}{2} \\
0 & 0 & -2 \sin (2 t) & \cos (2 t)
\end{array}\right) .
$$

Note that the matrix $M_{\mathbf{z}_{\alpha}}(t)$ does not depend of the particular periodic solution $\mathbf{x}\left(t, \mathbf{z}_{\alpha}\right)$. Since the matrix

$$
M^{-1}(0)-M^{-1}\left(p T_{1}\right)=\left(\begin{array}{cccc}
0 & 0 & 0 & 0 \\
0 & 0 & 0 & 0 \\
0 & 0 & 1-\cos \left(\frac{4 \sqrt{3} p \pi}{3}\right) & \frac{\sin \left(\frac{4 \sqrt{3} p \pi}{3}\right)}{2} \\
0 & 0 & -\sin \left(\frac{4 \sqrt{3} p \pi}{3}\right) & 1-\cos \left(\frac{4 \sqrt{3} p \pi}{3}\right)
\end{array}\right)
$$

satisfies the assumptions of statement (ii) of Theorem [ because the determinant

$$
\left|\begin{array}{cc}
1-\cos \left(\frac{4 \sqrt{3} p \pi}{3}\right) & \frac{\sin \left(\frac{4 \sqrt{3} p \pi}{3}\right)}{2} \\
-\sin \left(\frac{4 \sqrt{3} p \pi}{3}\right) & 1-\cos \left(\frac{4 \sqrt{3} p \pi}{3}\right)
\end{array}\right|=4 \sin ^{2}\left(\frac{2 \sqrt{3} p \pi}{3}\right) \neq 0,
$$

we can apply this theorem to the unperturbed system.

Now $\xi: \mathbb{R}^{4} \rightarrow \mathbb{R}^{2}$ is $\xi(X, Y, Z, W)=(X, Y)$. We calculate the function

$$
\mathcal{G}\left(X_{0}, Y_{0}\right)=\mathcal{G}(\alpha)=\xi\left(\frac{1}{p T_{1}} \int_{0}^{p T_{1}} M_{\mathbf{z}_{\alpha}}^{-1}(t) G_{1}\left(t, \mathbf{x}\left(t, \mathbf{z}_{\alpha}\right)\right) d t\right)
$$


and we obtain

$$
\left(\begin{array}{c}
\mathcal{F}_{1}\left(X_{0}, Y_{0}\right) \\
\mathcal{F}_{2}\left(X_{0}, Y_{0}\right)
\end{array}\right)=\left(\begin{array}{c}
\frac{1}{p T_{1}} \int_{0}^{p T_{1}} \frac{\sin (\sqrt{3} t)}{\sqrt{3}} F_{1}\left(t, \Delta_{1}(t), \Delta_{2}(t), 0,0\right) d t \\
\frac{1}{p T_{1}} \int_{0}^{p T_{1}} \cos (\sqrt{3} t) F_{1}\left(t, \Delta_{1}(t), \Delta_{2}(t), 0,0\right) d t
\end{array}\right) .
$$

Using

$$
F_{1}\left(t, \Delta_{1}(t), \Delta_{2}(t), 0,0\right)=\Delta_{1}(t) f_{1}\left(t, \Delta_{2}(t), 0\right)
$$

we obtain the functions given by (4). Then, by Theorem 6 we have that for every simple zero $\left(X_{0}^{*}, Y_{0}^{*}\right) \in V$ of the system of nonlinear functions

$$
\mathcal{F}_{1}\left(X_{0}, Y_{0}\right)=0, \quad \mathcal{F}_{2}\left(X_{0}, Y_{0}\right)=0,
$$

we have a periodic solution $(X, Y, Z, W)(t, \varepsilon)$ of the unperturbed system such that

$$
(X, Y, Z, W)(t, \varepsilon) \rightarrow\left(X_{0}^{*}, Y_{0}^{*}, 0,0\right) \quad \text { as } \varepsilon \rightarrow 0 .
$$

\section{Proof of the two COROLlaries}

Proof of Corollary 2. Under the assumptions of Corollary 2 the nonlinear equations (5) becomes

$$
\begin{aligned}
& \mathcal{F}_{1}\left(X_{0}, Y_{0}\right)=\frac{Y_{0}\left(3 X_{0}^{2}+Y_{0}^{2}\right)}{48}, \\
& \mathcal{F}_{2}\left(X_{0}, Y_{0}\right)=\frac{\left(3\left(\sqrt{3}-3 X_{0}\right) X_{0}^{2}-\left(\sqrt{3}+3 X_{0}\right) Y_{0}^{2}\right)}{24} .
\end{aligned}
$$

This system has the following real solution

$$
\left(X_{0}^{*}, Y_{0}^{*}\right)=\left(\frac{\sqrt{3}}{3}, 0\right)
$$

Moreover

$$
\operatorname{det}\left(\left.\frac{\partial\left(\mathcal{F}_{1}, \mathcal{F}_{2}\right)}{\partial\left(X_{0}, Y_{0}\right)}\right|_{\left(X_{0}, Y_{0}\right)=\left(\frac{\sqrt{3}}{3}, 0\right)}\right)=\frac{1}{384}
$$

check that this solution is simple. So, by Theorem 1 we only have one periodic solution of (11). This completes the proof of the corollary.

Proof of Corollary 4. Under the assumptions of Corollary 4 the nonlinear equations (6) becomes

$$
\begin{aligned}
& \mathcal{G}_{1}\left(Z_{0}, W_{0}\right)=\frac{\left(Z_{0}-1\right) W_{0}}{8}, \\
& \mathcal{G}_{2}\left(Z_{0}, W_{0}\right)=\frac{\left(4 Z_{0}\left(2+Z_{0}-2 Z_{0}^{2}\right)-W_{0}^{2}\left(1+2 Z_{0}\right)\right)}{32} .
\end{aligned}
$$


This system has the following four real solutions

$$
\left(Z_{0}^{*}, W_{0}^{*}\right)=\left(1, \pm \frac{2 \sqrt{3}}{3}\right),\left(Z_{0}^{*}, W_{0}^{*}\right)=\left(\frac{1-\sqrt{17}}{4}, 0\right)
$$

and

$$
\left(Z_{0}^{*}, W_{0}^{*}\right)=\left(\frac{1+\sqrt{17}}{4}, 0\right) .
$$

The solutions which differ in a sign are different initial conditions of the same periodic solution of the system (2) and

$$
\begin{aligned}
& \operatorname{det}\left(\left.\frac{\partial\left(\mathcal{G}_{1}, \mathcal{G}_{1}\right)}{\partial\left(Z_{0}, W_{0}\right)}\right|_{\left(Z_{0}, W_{0}\right)=\left(1, \pm \frac{2 \sqrt{3}}{3}\right)}=\frac{1}{32},\right. \\
& \operatorname{det}\left(\left.\frac{\partial\left(\mathcal{G}_{1}, \mathcal{G}_{1}\right)}{\partial\left(Z_{0}, W_{0}\right)}\right|_{\left(Z_{0}, W_{0}\right)=\left(\frac{1-\sqrt{17}}{4}, 0\right)}\right)=\frac{17-7 \sqrt{17}}{512}, \\
& \operatorname{det}\left(\left.\frac{\partial\left(\mathcal{G}_{1}, \mathcal{G}_{1}\right)}{\partial\left(Z_{0}, W_{0}\right)}\right|_{\left(Z_{0}, W_{0}\right)=\left(\frac{1+\sqrt{17}}{4}, 0\right)}\right)=\frac{17+7 \sqrt{17}}{512} .
\end{aligned}
$$

Therefore, by Theorem 3 we only have three periodic solutions of the perturbed dumbbell satellite. This completes the proof of the corollary.

\section{ApPendix: BASIC RESUlts ON AVERAGING THEORY}

In this appendix we present the basic result from the averaging theory that we shall need for proving the main results of this paper.

We consider the problem of the bifurcation of $T$-periodic solutions from differential systems of the form

$$
\dot{\mathbf{x}}(t)=G_{0}(t, \mathbf{x})+\varepsilon G_{1}(t, \mathbf{x})+\varepsilon^{2} G_{2}(t, \mathbf{x}, \varepsilon),
$$

with $\varepsilon=0$ to $\varepsilon \neq 0$ sufficiently small. Here the functions $G_{0}, G_{1}: \mathbb{R} \times \Omega \rightarrow$ $\mathbb{R}^{n}$ and $G_{2}: \mathbb{R} \times \Omega \times\left(-\varepsilon_{0}, \varepsilon_{0}\right) \rightarrow \mathbb{R}^{n}$ are $\mathcal{C}^{2}$ functions, $T$-periodic in the first variable, and $\Omega$ is an open subset of $\mathbb{R}^{n}$. The main assumption is that the unperturbed system

$$
\dot{\mathbf{x}}(t)=G_{0}(t, \mathbf{x}),
$$

has a submanifold of periodic solutions.

Let $\mathbf{x}(t, \mathbf{z}, \varepsilon)$ be the solution of the system (12) such that $\mathbf{x}(0, \mathbf{z}, \varepsilon)=\mathbf{z}$. We write the linearization of the unperturbed system along a periodic solution $\mathbf{x}(t, \mathbf{z}, 0)$ as

$$
\dot{\mathbf{y}}(t)=D_{\mathbf{x}} G_{0}(t, \mathbf{x}(t, \mathbf{z}, 0)) \mathbf{y}
$$


In what follows we denote by $M_{\mathbf{z}}(t)$ some fundamental matrix of the linear differential system (13), and by $\xi: \mathbb{R}^{k} \times \mathbb{R}^{n-k} \rightarrow \mathbb{R}^{k}$ the projection of $\mathbb{R}^{n}$ onto its first $k$ coordinates; i.e. $\xi\left(x_{1}, \ldots, x_{n}\right)=\left(x_{1}, \ldots, x_{k}\right)$.

We assume that there exists a $k$-dimensional submanifold $\mathcal{Z}$ of $\Omega$ filled with $T$-periodic solutions of (12). Then an answer to the problem of bifurcation of $T$-periodic solutions from the periodic solutions contained in $\mathcal{Z}$ for system (11) is given in the following result.

Theorem 6. Let $V$ be an open and bounded subset of $\mathbb{R}^{k}$, and let $\beta$ : $\mathrm{Cl}(V) \rightarrow \mathbb{R}^{n-k}$ be a $\mathcal{C}^{2}$ function. We assume that

(i) $\mathcal{Z}=\left\{\mathbf{z}_{\alpha}=(\alpha, \beta(\alpha)), \alpha \in \mathrm{Cl}(V)\right\} \subset \Omega$ and that for each $\mathbf{z}_{\alpha} \in \mathcal{Z}$ the solution $\mathbf{x}\left(t, \mathbf{z}_{\alpha}\right)$ of (12) is T-periodic;

(ii) for each $\mathbf{z}_{\alpha} \in \mathcal{Z}$ there is a fundamental matrix $M_{\mathbf{z}_{\alpha}}(t)$ of (13) such that the matrix $M_{\mathbf{z}_{\alpha}}^{-1}(0)-M_{\mathbf{z}_{\alpha}}^{-1}(T)$ has in the upper right corner the $k \times(n-k)$ zero matrix, and in the lower right corner $a(n-k) \times(n-k)$ matrix $\Delta_{\alpha}$ with $\operatorname{det}\left(\Delta_{\alpha}\right) \neq 0$.

We consider the function $\mathcal{G}: \mathrm{Cl}(V) \rightarrow \mathbb{R}^{k}$

$$
\mathcal{G}(\alpha)=\xi\left(\frac{1}{T} \int_{0}^{T} M_{\mathbf{z}_{\alpha}}^{-1}(t) G_{1}\left(t, \mathbf{x}\left(t, \mathbf{z}_{\alpha}\right)\right) d t\right) .
$$

If there exists $a \in V$ with $\mathcal{G}(a)=0$ and $\operatorname{det}((d \mathcal{G} / d \alpha)(a)) \neq 0$, then there is a T-periodic solution $\varphi(t, \varepsilon)$ of system (11) such that $\varphi(0, \varepsilon) \rightarrow \mathbf{z}_{a}$ as $\varepsilon \rightarrow 0$.

Theorem 6 goes back to Malkin [5] and Roseau [6], for a shorter proof see [1].

\section{ACKNOWLEDGEMEnTS}

This work has been partially supported by MICINN/FEDER grant number MTM2011-22587.

\section{REFERENCES}

[1] A. Buic̆ , J.P. FranÇOISE AND J. Llibre, Periodic solutions of nonlinear periodic differential systems with a small parameter, Communication on Pure and Applied Analysis 6 (2007), 103-111.

[2] M. T. de Bustos, J. L. G. Guirao, J. A. Vera and J. Vigo-Aguiar, Periodic orbits and $\mathcal{C}^{1}$-integrability in the planar Stark-Zeeman problem, J. of Mathematical Physics 53, 082701 (2012).

[3] J.L.G. Guirao, J. Llibre and J.A. Vera, Generalized van der Waals Hamiltonian: Periodic orbits and $C^{1}$ nonintegrability, Physical Review E 85 (2012), 036603.

[4] J.L.G. Guirao, J. Llibre And J.A. VerA, On the dynamics of the rigid body with a fixed point: periodic orbits and integrability, Nonlinear Dynamics, DOI: 10.1007/s11071-013-0797-8, 2013.

[5] I.G. MaLkin, Some problems of the theory of nonlinear oscillations, (Russian) Gosudarstv. Izdat. Tehn.-Teor. Lit., Moscow, 1956.

[6] M. Roseau, Vibrations non linéaires et théorie de la stabilité, (French) Springer Tracts in Natural Philosophy, Vol.8 Springer-Verlag, Berlin-New York, 1966.

[7] J.A. VERA, Dynamics of a triaxial gyrostat at a Lagrangian equilibrium of a binary asteroid, Astrophysics and Space Science 323(4) (2009), 375-382 
[8] J. A. VERA, On the dynamics of a gyrostat on Lagrangian equilibria in the three body problem, Multibody System Dynamics 23 (3) (2010), 263-291.

${ }^{1}$ Departamento de Matemática Aplicada y Estadística. Universidad Politécnica de Cartagena, Hospital de Marina, 30203-Cartagena, Región de Murcia, Spain.Corresponding Author-

E-mail address: juan.garcia@upct.es

2 Centro Universitario de la Defensa. Academia General del Aire. Universidad Politécnica de Cartagena, 30720-Santiago de la Ribera, Región de Murcia, Spain.

E-mail address: juanantonio.vera@cud.upct.es

${ }^{3}$ Department of Mathematical Sciences, University of Wisconsin-Milwaukee, MilWAUKeE, WI 53201-0413, USA.

E-mail address: wade@uwm.edu 